\title{
Conectivismo y diseño instruccional: ecología de aprendizajes para la universidad del siglo XXI en México
}

\author{
Connectivism and instructional design: learning ecology \\ for the 21st century university in Mexico
}

\author{
Anna Beatriz Prado*
}

Recibido: 28 de mayo de 2020 Aceptado: 15 de junio de 2020 Publicado: 31 de enero de 2021

To cite this article: Prado-Rodríguez, A. B. (2021). Conectivismo y diseño instruccional: ecología de aprendizajes para la universidad del siglo XXI en México. Márgenes, Revista de Educación de la Universidad de Málaga, 2 (1), 4-20

DOI: https://doi.org/10.24310/mgnmar.v2i1.9349

\section{RESUMEN}

El presente ensayo ofrece una reflexión sobre las nuevas perspectivas que presenta la universidad del siglo XXI en México, en específico los procesos de enseñanza y aprendizaje, gracias a una amplia revisión bibliográfica. Actualmente, se habla de la Sociedad del Conocimiento concebida como una aldea global interconectada por diversas tecnologías que facilitan el intercambio de información, influyendo en la toma de decisiones individual y colectivamente. Las Instituciones de Educación Superior (IES) se ven afectadas o influenciadas por esta situación, pues son las encargadas de formar individuos íntegros y profesionales, debiendo adaptar el proceso enseñanza-aprendizaje para los nuevos objetivos que exige la sociedad globalizada.

El documento se divide en cuatro apartados, inicia con una breve explicación sobre las características de la educación y el aprendizaje del siglo XXI en la educación superior; continua con una descripción de los principios teóricos del conectivismo que facilita la comprensión del nuevo aprendizaje; después, retoma la nueva organización para la enseñanza a través el diseño instruccional mediado por las herramientas tecnológicas; posteriormente, reconoce la situación actual de la universidad mexicana ante los retos que presenta el siglo XXI, donde se identifica la necesidad de una ecología de aprendizaje; y concluye, estableciendo sus perspectivas.

Palabras clave: conectivismo; diseño instruccional; ecología de aprendizajes; educación superior

\section{ABSTRACT}

This essay offers a reflection on the new perspectives presented by the university of the 21st century in Mexico, specifically the processes of teaching and learning, thanks to an extensive bibliographical review. Currently, we talk about the Knowledge Society conceived as a global village interconnected by various technologies that facilitate the exchange of information, influencing individual and collective decision-making. Higher Education Institutions (HEIs) are affected or influenced by this situation, since they are responsible for training upright and professional individuals, having to adapt the teaching-learning process to the new objectives required by globalize society. 
The document is divided into four sections. It begins with a brief explanation of the characteristics of education and learning in the 21st century in higher education; it continues with a description of the theoretical connectivism principles that facilitate the understanding of new way of learning; therefore, goes on to discuss the new teaching organization through instructional design mediated by technological tools; afterwards, recognizes the current situation of Mexican universities in the face of the challenges presented by the 21st century, identifying the need of a learning ecology; and ends with the consideration of their perspectives.

Keywords: connectivism; instructional design; learning ecology; higher education

\section{EDUCACIÓN SUPERIOR Y APRENDIZAJE EN EL SIGLO XXI}

Desde su surgimiento, la educación se ha visto afectada por los cambios sociales, económicos y políticos que experimenta la sociedad, destacando últimamente los tecnológicos y científicos (OECD, 2015). Gracias a ellos, la educación siempre se propone adaptarse a estos cambios para ayudar a la sociedad mediante los ajustes realizados a las diferentes disciplinas que la sustentan, tales como psicología educativa, pedagogía, didáctica, investigación educativa, etc. Actualmente, en la era digital, la educación está adaptándose a las exigencias y retos que se manifiestan, siendo el más destacado el cambio vertiginoso de la información y comunicación (García Aretio, Ruíz, Quintana, García Blanco, y García Pérez, 2009).

En los últimos 30 años, las tecnologías digitales han tenido un desarrollo asombroso en la sociedad, esto inicia con la accesibilidad de la computadora, aplicación de software de ofimática, popularización del internet y el uso del correo electrónico, influyendo en todas las áreas de la sociedad implicando a la educación; por consiguiente, las tecnologías son incluidas en la educación bajo la perspectiva de calidad, realizando ajustes al proceso enseñanza-aprendizaje para afrontar la nueva creación de contenidos educativos digitales que comparten conocimiento, tecnologías e información (OREALC, 2013).

La conferencia mundial sobre educación superior (2009) en su comunicado establece que la educación superior está comprometida a reconocer la necesidad de tres conceptos importantes acceso, equidad y calidad, explicados en los siguientes puntos (UNESCO, 2010, p. 3):

11. [...] La educación superior debe ampliar la formación de docentes, tanto inicial como en el empleo, con planes y programas de estudios que den a los docentes la capacidad de dotar a sus alumnos de los conocimientos y las competencias que necesitan en el siglo XXI. Este objetivo exigirá nuevos enfoques, como por ejemplo el uso del aprendizaje abierto y a distancia y de las tecnologías de la información y la comunicación (TIC).

12. La formación de expertos en planificación educativa y la realización de investigaciones pedagógicas con el fin de mejorar las estrategias didácticas contribuyen también a la consecución de los objetivos de la educación para todos.

A finales del siglo pasado apareció el concepto de la sociedad de la información y conocimiento, gracias al surgimiento de las Tecnologías de la Información y Comunicación (TIC), que distingue la presencia de factores simbólicos y conocimiento (cognición, creatividad, conocimiento e información) como parte del sistema de producción; siendo el conocimiento lo que contribuye 
a la productividad de un país (Barojas, Sierra, y Martínez, 2006). Dicha sociedad surge con los avances científicos y tecnológicos dentro de un marco socioeconómico globalizado reflejando el mundo del siglo XXI, encontrándose sustentada en cuatro pilares: la libertad de expresión, el acceso universal a la información y al conocimiento, el respeto a la diversidad cultural y lingüística, así como la educación de calidad para todos.

Villalonga (2015) comenta que, con el surgimiento de la sociedad del conocimiento, el uso múltiple del internet y la aplicación de las tecnologías digitales en la educación han iniciado una nueva etapa histórica dentro la educación a distancia; de manera que, al apropiarse de las TIC la educación a distancia, a través de las Instituciones de Educación Superior (IES), ha creado nuevas modalidades educativas semipresencial o dual (b-learning) y en línea o virtual (e-learning). Fortaleciendo lo anterior, la meta 4.3 del documento Educación 2030, dentro de sus estrategias indicativas, dicta la elaboración de políticas y programas para la impartición de educación superior a distancia de calidad, que aproveche la tecnología, incluyendo el uso de internet, cursos abiertos en línea con participación masiva facilitando su accesibilidad (UNESCO, 2016).

Según el Banco Interamericano de Desarrollo (2012), citado por la Oficina de Representación de Educación para América Latina y del Caribe (OREALC, 2013), han incrementado con rapidez la tasa de incorporación de tecnologías y conectividad, a pesar del largo recorrido por asegurar el acceso equitativo y universal. En este sentido, la Comisión Interamericana (2003) estableció planes de acción para la educación por la agenda de conectividad para las Américas, relacionándolos con la solución de "la inequidad en el acceso al sistema educativo, la baja calidad y la falta de uniformidad en cuanto al contenido educativo y métodos de enseñanza para los ciudadanos en diferentes zonas y regiones geográficas de los países” (p. 34), obligando a los gobiernos a comenzar a trabajar desde el desarrollo de políticas públicas hasta la creación de infraestructura que facilite la conectividad para la población.

Inclusive, la OREALC (2013) plantea que las políticas públicas deben propiciar las nuevas experiencias de aprendizaje a través de la compra de equipos, inversión en capacitación y formación, recursos educativos innovadores, entre otras. El éxito de la propuesta para la construcción del nuevo paradigma educativo, sugerido por la OREALC (2013), requiere de tres condiciones:

a. Centralidad de los estudiantes busca la "personalización de la oferta educativa”, ubicando al educando en un plano central con sus propias características, intereses, condiciones, expectativas y potencial, para transformarse en un espacio de desarrollo e integración.

b. Alineamiento de los requerimientos de la sociedad del conocimiento estrechamente relacionado con las necesidades del medio, permitiendo a los alumnos su contribución creativa en el desarrollo, comunicación y construcción del conocimiento, en apoyo de una sociedad inclusiva, equitativa y participativa.

c. Integralidad e implementación sistémica debe presentar claridad en su conformación, de tal forma que sus procesos, integrantes y resultados respondan integralmente al nuevo reto.

La tecnología educativa es una herramienta indispensable e inseparable para la sociedad del conocimiento, a través de ella los participantes logran el aprendizaje, categorizado en formal e informal. Actualmente, los alumnos viven la tecnología como parte de su entorno cotidiano y 


\section{ESTUDIOS Y ENSAYOS}

aprenden de manera informal, es decir fuera del salón de clases; por consiguiente, los docentes deben promover el aprendizaje formal dentro del aula, para desarrollar su creatividad mediante el manejo de las TIC. Es por ello que, la sociedad del conocimiento propone resignificar las prácticas educativas a través de seis nuevos componentes para alcanzar la meta de una educación para todos.

\begin{tabular}{|c|c|}
\hline Personalización & $\begin{array}{l}\text { - Innovaciones educativas (TIC) deben incorporar el proyecto de vida del estudiante } \\
\text { como parte contitutiva y cuncial de la experiencia educativa. }\end{array}$ \\
\hline $\begin{array}{l}\text { Foco en los resultados de } \\
\text { aprendizaje }\end{array}$ & $\begin{array}{l}\text {-Innovaciones educativas (TIC) permiten acceder al conocimiento, comunicarlo rápida y } \\
\text { eficázmente, además de evaluar el aprendizaje y su proceso. }\end{array}$ \\
\hline $\begin{array}{c}\text { Ampliación de tiempos y } \\
\text { espacios para el aprendizaje }\end{array}$ & $\begin{array}{l}\text { - Innovaciones educativas (TIC) facilitan la ubicuidad de las experiencias educativas de } \\
\text { manera ilimitada en tiempo y espacio. }\end{array}$ \\
\hline $\begin{array}{l}\text { Nuevas experiencias de } \\
\text { aprendizaje }\end{array}$ & $\begin{array}{l}\text { - Innovaciones educativas (TIC) desarrollan nuevas experiencias de aprendizajes al } \\
\text { incorporar nuevos diseños instruccionales, estrategias y recursos educativos. }\end{array}$ \\
\hline $\begin{array}{l}\text { Construcción colaborativa de } \\
\text { conocimientos }\end{array}$ & $\begin{array}{l}\text {-Innovaciones educativas (TIC) permiten una mejor conección de experiencias de } \\
\text { aprendizaje con la comunidad. }\end{array}$ \\
\hline $\begin{array}{l}\text { Gestión del conocimiento } \\
\text { basado en la evidencia }\end{array}$ & $\begin{array}{l}\text { - Sistemas tecnológicos (TIC) enriquesen los sistemas educativos con los registros de las } \\
\text { acciones y progresos de la experiencia de aprendizaje. }\end{array}$ \\
\hline
\end{tabular}

Ilustración 1. Componentes de nuevas prácticas educativas para la sociedad del conocimiento. Elaboración propia a partir de (OREALC, 2013)

Previamente, Roldan (2013) propone que la educación de este siglo debe contar con infraestructura en recursos humanos experto de las diferentes áreas educativas y manejo de tecnología, por ende, se habla de una economía del conocimiento pues en ella se encuentran los cimientos de la educación de calidad e investigación, generadora de innovación y desarrollo.

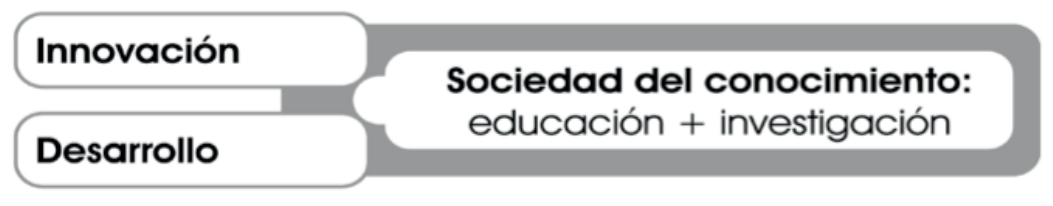

Ilustración 2. Elementos de la economía del conocimiento. A partir de Roldán (2013, p. 81) 
Actualmente, la mayoría de los individuos adoptan los teléfonos celulares, las redes sociales, computadoras e internet como medio de comunicación que a su vez facilita su propia enseñanza y aprendizaje, con dichas herramientas tecnológicas pueden encontrar, sintetizar e integrar información de variados recursos. Sin embargo, pocos poseen la llamada destreza de literacidad de información que involucra la gestión y administración de la información incluyendo el trabajo colaborativo en redes, considerada como la habilidad de la futura sociedad por la American Library Association (Siemens, 2008). Por lo tanto, las IES juegan un papel importante en la consolidación y fortalecimiento de esta destreza de literacidad iniciando en el ámbito académicoeducativo de los alumnos para ejercerla posteriormente en lo laboral.

Por otro lado, OECD (2015) indica que dentro de la educación superior existe el "movimiento de educación abierta” (p. 41), aquella donde los Open Educational Resorces (OER) o recursos educativos abiertos irrumpen gracias a la mediación de las nuevas tecnologías emergentes y su objetivo es romper las barreras de enseñanza-aprendizaje, incorporando las nuevas tecnologías digitales para compartir los OER en la comunidad educativa global. Por ende, el e-learning o educación virtual en conjunto con las TIC y los OER permiten la democratización del conocimiento, especialmente en la actual economía del conocimiento pues esta exige calidad educativa (OECD, 2015). En síntesis, la economía del conocimiento es la nueva forma de aprendizaje social, donde "el alumno juega un rol diferente, al trabajar a su paso e interactuar con otros para intercambiar perspectivas, colaborar y actualizar dinámicamente contenidos para crear conocimiento" (pp. 52), esta red de estudiantes se transforma en la red de creación de conocimiento según la teoría del conectivismo de Siemens y Downes (OECD, 2015).

\section{CONECTIVISMO: UNA EXPLICACIÓN TEÓRICA PARA EL NUEVO APRENDIZAJE}

En las últimas dos décadas la tecnología ha reestructurado nuestras vidas, la comunicación y el aprendizaje, actualmente el conocimiento crece vertiginosamente midiéndose en meses. Ahora se accede a las diversas fuentes del conocimiento a través de la conexión infinita de datos, información en bruto y sin procesar, teniendo implicaciones en la forma de aprender y de generar interacción con el conocimiento; por ello, surge un nuevo enfoque teórico educativo dentro de la era digital que actualmente vivimos, el conectivismo

Uno de los fundadores de este enfoque teórico es George Siemens, quien define al conectivismo como "un ciclo donde el conocimiento personal se compone de una red, la cual alimenta a organizaciones e instituciones, las que a su vez retroalimentan a la red, proveyendo nuevo aprendizaje para los individuos" (Siemens, 2004, p.7), donde es predominante tener la destreza para aprender en estas conexiones. Otro precursor del conectivismo es Stephen Downes, quien lo fundamenta con cuatro conceptos esenciales que continuación se intentan sintetizar:

a. El conocimiento es "el conjunto de conexiones entre entidades" (Downes, 2012, p. 9). En los individuos se establecen conexiones neuronales, en las sociedades las conexiones ocurren entre individuos y herramientas tecnológicas, si el conocimiento sucede en la red debe haber una identificación de patrones en las conexiones e interacciones. 


\section{E S T U D I S Y E N SAYOS}

b. El aprendizaje es definido como "la creación o eliminación de conexiones entre las entidades o el ajuste de sus fortalezas” (Downes, 2012, p. 9). Es decir, la creación de conexiones adaptadas se alcanza por los mecanismos de similitud, contigüidad, retroalimentación y armonía, entre otros, que trabajan individual o conjuntamente siendo irreductiblemente complejos.

c. La comunidad "describe las condiciones exitosas o efectivas de las redes que permiten aprender, se pueden adaptar o ajustar, evitan su estancamiento o muerte” (Downes, 2012, p.9). Toda red debe contar con condiciones semánticas y físicas, que favorezcan su dinámica y distribución, de igual forma dichas condiciones están conformadas por los elementos de autonomía, diversidad, apertura y conectividad o interactividad. Si las redes favorecen estas condiciones aseguran su desarrollo y crecimiento sin importar el tipo de comunidad de aprendizaje, tecnologías o sociedades.

d.El lenguaje "contiene el conocimiento" y es "el acto de comunicar entre una entidad y otra" (Downes, 2012, p. 19), o sea, este es concebido como una entidad constituida con su propia organización interna, sin embargo, es inseparable de la comunidad que lo creó. El lenguaje es el reflejo de lo aprendido y experienciado, por lo que, no constituye el aprendizaje ni las experiencias, en sí.

Siemens (2008) retoma la propuesta de Ertmer y Newby para responder las cinco preguntas que permiten distinguir las teorías de aprendizaje, él lo hace con el conectivismo de manera fundamentada y organizada para sustentar su propuesta teórica.

Tabla 1. Teoría del Conectivismo

\begin{tabular}{|c|c|c|c|c|}
\hline $\begin{array}{l}\text { ¿Cómo ocurre el } \\
\text { aprendizaje? }\end{array}$ & $\begin{array}{l}\text { ¿Qué factores } \\
\text { influyen en el } \\
\text { aprendizaje? }\end{array}$ & $\begin{array}{l}\text { ¿Qué papel } \\
\text { desempeña la } \\
\text { memoria? }\end{array}$ & $\begin{array}{c}\text { ¿Cómo } \\
\text { ocurre la } \\
\text { transferencia? }\end{array}$ & $\begin{array}{c}\text { ¿Qué tipo el } \\
\text { aprendizaje explica la } \\
\text { teoría? }\end{array}$ \\
\hline $\begin{array}{l}\text { Distribuido dentro de } \\
\text { la red, social, mejorado } \\
\text { tecnológicamente, } \\
\text { reconociendo e } \\
\text { interpretando patrones. }\end{array}$ & $\begin{array}{l}\text { Diversidad en la } \\
\text { red, fuerza de las } \\
\text { conexiones. }\end{array}$ & $\begin{array}{l}\text { Patrones adaptati- } \\
\text { vos, representación } \\
\text { del estado actual } \\
\text { existentes en la red. }\end{array}$ & $\begin{array}{c}\text { Conexión } \\
\text { (aumentando) } \\
\text { de nodos o } \\
\text { redes. }\end{array}$ & $\begin{array}{c}\text { Aprendizaje complejo, } \\
\text { cambio rápido } \\
\text { de información, } \\
\text { diversos recursos de } \\
\text { conocimiento. }\end{array}$ \\
\hline
\end{tabular}

Fuente: a partir de Siemens, (2008) (p. 11)

De tal forma que el conectivismo, de acuerdo con Siemens (2004), está constituido de manera general por cuatro principios integradores:

1. La teoría del caos, reconoce la conexión del todo con el todo donde el significado del conocimiento y el orden se encuentra encriptado.

2. La teoría de redes, donde la red está conformada por conexiones entre entidades llevando a cabo interacciones para crear aprendizajes y conocimientos.

3. La complejidad relacionada con la toma de decisiones respecto a la información cambiante, desarrollando una habilidad crítica sobre su elección. 


\section{EST U D OS Y ENSAYOS}

4. Aprendizaje (auto-organización) es la habilidad de reconocimiento y ajuste de cambio de patrones.

La teoría conectivista reconoce en el aprendizaje un conocimiento aplicable, fuera del individuo que enlaza información especializada con otras conexiones para incrementarlo, todo ello gracias al desarrollo de la habilidad crítica para tomar decisiones; a continuación, se describe sintéticamente sus preceptos específicos.

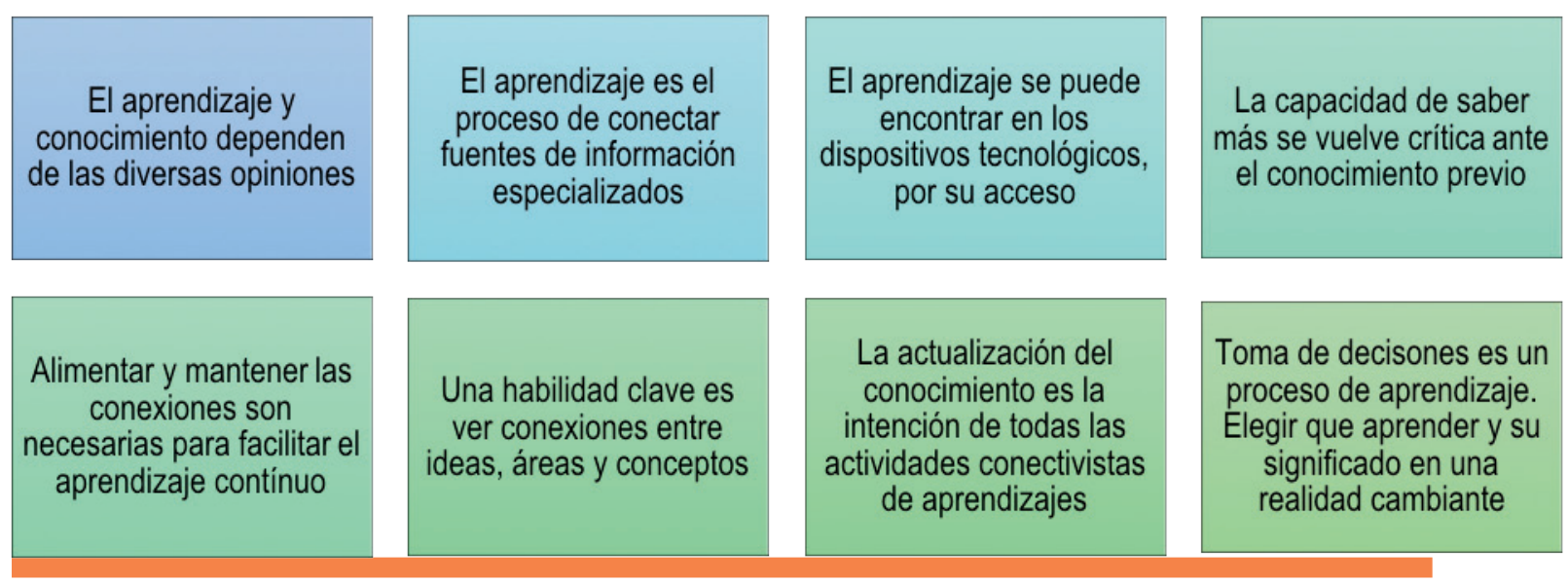

Ilustración 3. Principios del conectivismo. Elaboración propia a partir de Siemens (2004) (p. 6-7)

En esta teoría existe una economía del conocimiento que contempla cinco componentes, el conocimiento, la base de datos, el flujo de información, las personas conectadas y el contexto adecuado para procurar el aprendizaje. En otras palabras, el conocimiento se encuentra dentro de la base de datos, mientras que el flujo de información permite crearlo, preservarlo y utilizarlo por las personas conectadas en el contexto adecuado, como una ecología organizacional.

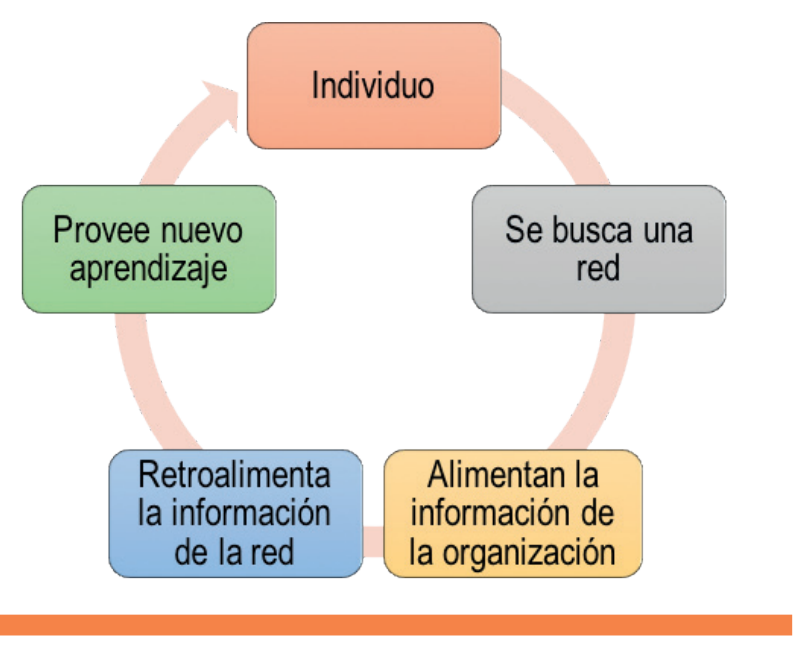

Ilustración 4. Ciclo del aprendizaje en red. Elaboración propia a partir de Rodríguez y Molero (2009, p. 77)
De esta manera el conocimiento es un proceso, según Bartolomé (2011), al percibirlo como una interpretación certera de la realidad, adquiriendo dos concepciones interesantes como producto de la negociación en el grupo-ante el individuo y como un continuo cambiante (dinámico). Mientras que Ovalles (2014) establece la distinción entre conocimiento y aprendizaje, el primero es un patrón específico de relaciones y el segundo es la creación de nuevas conexiones, así como la habilidad crítica para manipular redes o patrones existentes. Ambos, incluyen la tecnología como parte de la distribución de cognición y conocimiento, que es resguardada en las conexiones formadas por individuos o base de datos. 
Ahora bien, en la era digital el ser humano adquiere conocimiento al participar en redes sociales mediadas por tecnología y el aprendizaje es visto como una red donde los conceptos pueden ser considerados dominios o ejes de conocimiento, por consiguiente, conectivismo equivale aprendizaje en red y para optimizarlo hay que identificar de manera crítica las propiedades de las redes eficientes (Vadillo, 2011 y Bartolomé, 2011).

El conectivismo sugiere que el docente proporciona solo una guía de lecturas, conceptos, materiales didácticos mediante herramientas digitales y multimedia, entre otros, o sea no tiene el control total sobre el proceso de enseñanza-aprendizaje; mientras que, el alumno tiene la oportunidad de elegir su proceso, mediante la construcción de sus propios recursos, involucrándose activamente y pre-enseñándose, de esta manera demuestra su comprensión y aprendizaje.

Otra característica de esta teoría es que, la educación debe ser abierta, con accesibilidad de recursos, aumento de opciones tecnológicas complejas, con un diálogo continuo entre los modelos pedagógicos y docentes; es aquí donde, el rol del diseñador instruccional participa abiertamente como el educador del docente y de manera estrecha trabaja con la facultad, los diseñadores de materiales y el personal técnico (Siemens, 2008). En este caso, el diseñador instruccional tiene la tarea de traducir en un lenguaje de fácil comprensión para el docente el uso y aplicación de los sitios de recursos educación abierta, las herramientas de comunicación, los contenidos colaborativos con opción de filtrado o creación, así como del aprendizaje en red.

Por último, bajo este enfoque teórico el término de ecología de aprendizajes remplaza al de diseño instruccional y lo define como "un sistema en continua evolución” (Gutiérrez, 2012, p. 116). Donde las herramientas sincrónicas y asincrónicas, al igual que las de la web 2.0 y 3.0 permiten la gestión del conocimiento, acceso a la información, comunicación y construcción de redes de contacto, una vez integrados crean los entornos de aprendizaje basados en redes (Bartolomé, 2011).

\section{DISEÑO INSTRUCCIONAL: UNA NUEVA ORGANIZACIÓN PARA LA ENSEÑANZA}

Ahora las TIC son parte de la vida cotidiana además aplicadas en otros ámbitos, la educación las utiliza para promover la enseñanza y facilitar el aprendizaje. Estas herramientas tecnológicas son grandes aliadas para el desarrollo de la instrucción dentro y fuera del aula, el diseño instruccional no puede ser concebido sin la tecnología educativa. Es pertinente recordar el origen del diseño instruccional sustentado en la teoría del condicionamiento operante de B.F. Skinner, refiere que toda conducta debe ser observable y medible, este fue retomado posteriormente por la educación con su máquina de la enseñanza la cual establece una instrucción programada para observar y evaluar la respuesta del estudiante. Con ello se crea el vínculo inseparable entre el diseño instruccional y la tecnología.

En el siglo pasado se consolido la teoría del aprendizaje con la psicología de la instrucción o diseño instruccional, estableciendo la existencia de tres paradigmas el primero enfocado en la consolidación de la respuesta (conductismo), el segundo basado en la adquisición del conocimiento (cognitivismo), el tercero sustentado en la elaboración del conocimiento (constructivismo) y, actualmente, surgió uno nuevo fundamentado por el aprendizaje en redes (conectivismo). 


\section{ESTUDIOS Y ENSAYOS}

Tabla 2. Teorías del Aprendizaje y sus características

\begin{tabular}{|c|c|c|c|}
\hline \multicolumn{4}{|c|}{ TEORÍAS DEL APRENDIZAJE } \\
\hline CONDUCTISMO & COGNITIVISMO & CONSTRUCTIVISMO & CONECTIVISMO \\
\hline $\begin{array}{c}\text { Enseñanza programada y procesos } \\
\text { lineales }\end{array}$ & $\begin{array}{l}\text { Aprendizaje significati- } \\
\text { vo (aprendizaje previo } \\
\text { y modificación de es- } \\
\text { tructuras cognitivas) }\end{array}$ & $\begin{array}{l}\text { Construcción } \\
\text { del aprendizaje } \\
\text { (aprendizajes previos } \\
\text { e interpretación de la } \\
\text { información) }\end{array}$ & $\begin{array}{l}\text { Aprendizaje en redes, } \\
\text { es producto de la era } \\
\text { digital (conexiones } \\
\text { entre usuarios y su } \\
\text { retroalimentación) }\end{array}$ \\
\hline Información en pequeñas unidades & $\begin{array}{c}\text { Métodos educativos } \\
\text { encauzados a } \\
\text { memorizar, entender } \\
\text { y recordar los } \\
\text { conocimientos } \\
\text { para desarrollar } \\
\text { las capacidades } \\
\text { intelectuales }\end{array}$ & $\begin{array}{c}\text { Participación activa } \\
\text { del alumno }\end{array}$ & $\begin{array}{c}\text { El aprendizaje } \\
\text { se da fuera del } \\
\text { individuo, además } \\
\text { de ser almacenado } \\
\text { y manipulado por la } \\
\text { tecnología. }\end{array}$ \\
\hline $\begin{array}{l}\text { Diseño de actividades con respuesta } \\
\text { del alumno }\end{array}$ & $\begin{array}{l}\text { Recursos educativos } \\
\text { informáticos } \\
\text { (hipertextuales e } \\
\text { hipermediales) }\end{array}$ & $\begin{array}{l}\text { Los recursos didác- } \\
\text { ticos se enfatizan en } \\
\text { entorno de apren- } \\
\text { dizaje y los alumnos } \\
\quad \text { (hipermedios) }\end{array}$ & $\begin{array}{c}\text { Elaboración de } \\
\text { materiales educativos } \\
\text { informáticos valorando } \\
\text { lo que se aprende }\end{array}$ \\
\hline $\begin{array}{l}\text { Planifıcación del refuerzo en } \\
\text { materiales didácticos (diseño) }\end{array}$ & $\begin{array}{l}\text { Los recursos educa- } \\
\text { tivos informáticos re- } \\
\text { presentan los procesos } \\
\text { cognitivos }\end{array}$ & $\begin{array}{l}\text { Diseño con poco } \\
\text { contenido, enlaces } \\
\text { con referencias, } \\
\text { recursos y } \\
\text { herramientas. }\end{array}$ & $\begin{array}{l}\text { Se basa en los } \\
\text { fundamentos de } \\
\text { la sociedad del } \\
\text { conocimiento }\end{array}$ \\
\hline $\begin{array}{c}\text { Diseño de cursos y recursos } \\
\text { didácticos que presentan } \\
\text { información de manera gradual, } \\
\text { incrementan su complejidad, con } \\
\text { cadena de respuestas y reforzadores }\end{array}$ & $\begin{array}{l}\text { Diseño de recursos } \\
\text { educativos con con- } \\
\text { tenidos organizados } \\
\text { jerárquicamente }\end{array}$ & $\begin{array}{l}\text { Enfatiza el aprendizaje } \\
\text { mediante la resolu- } \\
\text { ción de problemas y } \\
\text { situaciones de apren- } \\
\text { dizaje colaborativo }\end{array}$ & $\begin{array}{l}\text { La instrucción se dise- } \\
\text { ña conforme a la visión } \\
\text { de un aprendizaje inte- } \\
\text { gral, completo, coope- } \\
\text { rativo y colaborativo }\end{array}$ \\
\hline Estructura rígida y lineal & Navegabilidad no lineal & $\begin{array}{l}\text { Navegación } \\
\text { compuesta }\end{array}$ & Conexiones por redes \\
\hline
\end{tabular}

Fuente: Elaboración propia a partir de CODAES (2015, pp. 10-11)

La tecnología educativa es reconocida como una herramienta en los procesos instructivos que facilita el flujo de la información y/o conocimiento; sin embargo, esta por sí sola no forma a los estudiantes, se requiere de especialistas en educación para planificar el proceso enseñanza-aprendizaje de calidad (García Aretio, et.al., 2009), quienes se encargan de optimizar la aplicación e interactividad de las herramientas tecnológicas gracias al diseño instruccional (Gómez, 2013). 


\section{ESTUDIOS Y ENSAYOS}

Por consiguiente, la sociedad del conocimiento promueve el desarrollo e incorporación de una pedagogía virtual con educación de calidad; esta pedagogía se lleva a cabo mediante la aplicación de las TIC, teniendo como elemento central al diseño instruccional para asegurar su funcionamiento mediante la sistematización y ordenamiento de necesidades, establecimiento de objetivos y metas, conocimientos de contenidos, diseño de materiales y actividades que evidencian el aprendizaje, establecer estrategias cognitivas y habilidades comunicativas, además de la evaluación del proceso para la futura toma de decisiones en la enseñanza-aprendizaje (García Cabrero, Márquez, Bustos, Miranda y Espíndola, 2008) a través de la propuesta siguiente.

Al incorporarse las herramientas tecnológicas en la educación surgen diferentes modalidades educativas. Para Gómez (2013) la educación en línea es aquella que emplea programas a distancia mediados por las TIC. Mientras que, para García Aretio, et.al. (2009) la educación a distancia o virtual se concibe bajo la "idea de ruptura" (p. 20) con la presencialidad física y la secuencialidad lineal de la información, apoyándose de las tecnológicas en comunicación síncrona y asíncrona, originando procesos de construcción de conocimiento completamente diferentes. Por otro lado, la OECD (2015) asegura su diferencia al definir e-learning como "el uso de las TIC para promover y mejorar el aprendizaje en la educación superior o terciaria” (p. 42), y utiliza como su sinónimo la palabra virtual.

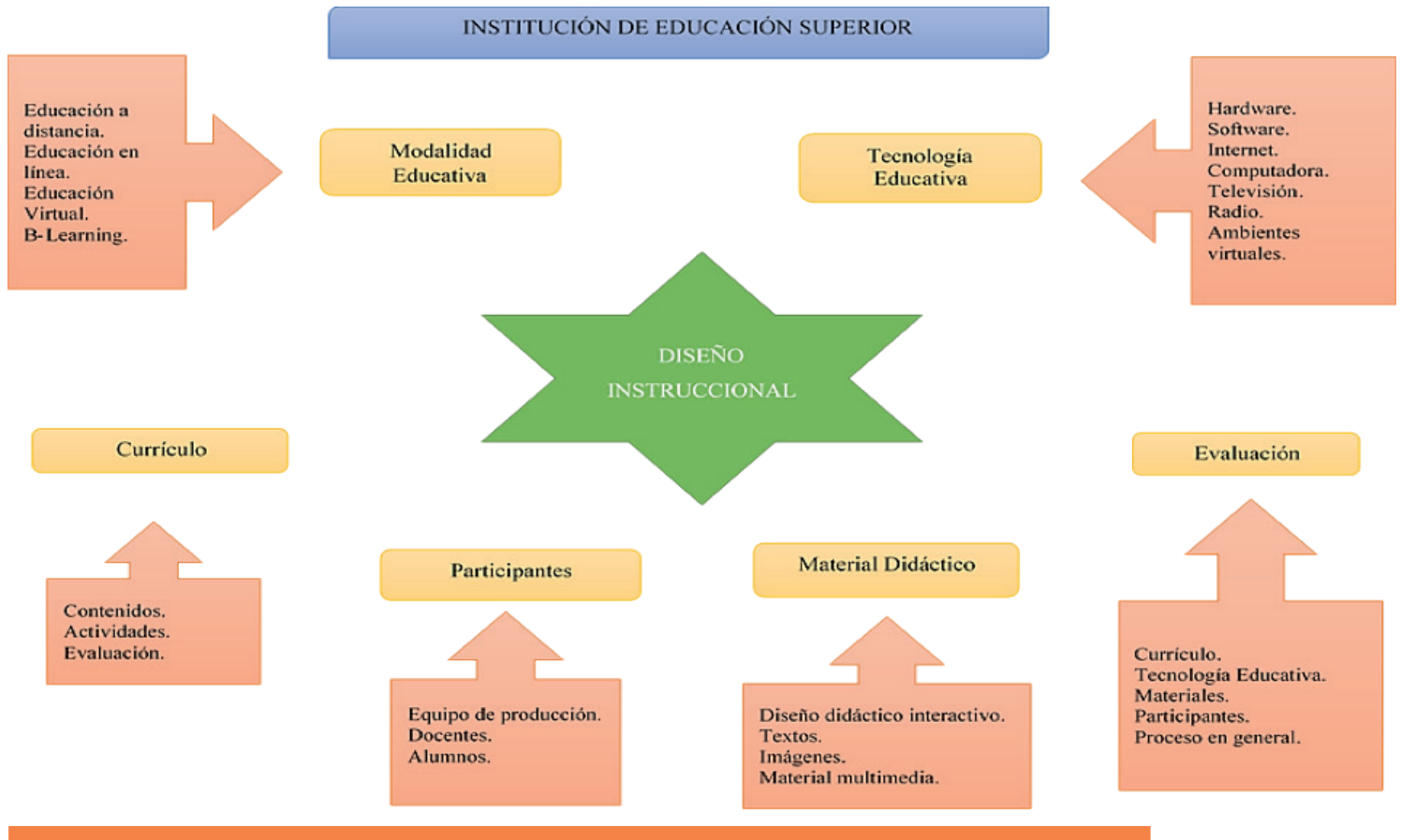

Ilustración 5. Componentes de un diseño instruccional. Elaboración propia (2019)

Esta modalidad educativa se constituye por los principios de apertura (acceso a la educación), flexibilidad (relacionado con el tiempo, espacio, aprendizaje individual, etc.), democratización (acerca la educación a todos), interactividad (comunicación multidireccional con trabajo colaborativo) y actividad consustancial a toda propuesta educativa (promotora del aprendizaje) (García Aretio, et.al., 2009). 


\section{ESTUDIOS Y ENSAYOS}

Como se ha establecido con anterioridad, la tecnología educativa es una herramienta poderosa que facilita al estudiante el desarrollo de sus capacidades y destrezas gracias al acceso de la información y el conocimiento, permitiéndole "aprender a aprender" con el internet, la multimedia, realidad virtual, gamificación, entre otras (Alismail y McGuire, 2015), reconociendo en los medios de comunicación síncrona y asíncrona como herramientas que favorecen la productividad y colaboración entre los participantes (Keeler, 2008). A continuación, se describen las herramientas de tecnología educativa más utilizadas en la modalidad educativa en línea.

Tabla 3. Tecnología educativa y sus características

\begin{tabular}{|c|c|}
\hline Nombre & Características \\
\hline \multirow[t]{3}{*}{ Computadora } & 1. Es la más conocida. \\
\hline & 2. Facilita el alcance de objetivos de aprendizaje. \\
\hline & 3. Instrumento mediador de capacidades de orden superior. \\
\hline \multirow{6}{*}{$\begin{array}{l}\text { Entorno Virtual de Aprendizaje (EVA) o } \\
\text { Ambiente Virtual de Aprendizaje (AVA) }\end{array}$} & 1. Es el aula de la educación en línea. \\
\hline & $\begin{array}{l}\text { 2. Usa la computadora y un sistema de comunicación (síncrona } \\
\text { y asíncrona). }\end{array}$ \\
\hline & $\begin{array}{l}\text { 3. La comunicación asincrónica (interacción en diferido) es rele- } \\
\text { vante en los procesos de comunicación e interacción. }\end{array}$ \\
\hline & 4. Favorece la flexibilidad comunicativa \\
\hline & 5. Crea materiales informáticos de enseñanza-aprendizaje. \\
\hline & 6. Soluciona problemas de espacio temporales. \\
\hline \multirow{5}{*}{$\begin{array}{l}\text { Curso en línea masivo y abierto (MOOC) } \\
\qquad \text {-siglas en inglés- }\end{array}$} & 1. Surge en el 2008. \\
\hline & 2. Masivo no existe un número límite de alumnos inscritos. \\
\hline & 3. En línea desarrollado en la web y su monitoreo no es obligatorio. \\
\hline & $\begin{array}{l}\text { 4. Abierto no se condiciona el acceso a quien ingresa, es gratuito } \\
\text { y abierto, pero si se desea tutoría o certificación tiene costo. }\end{array}$ \\
\hline & 5. Curso cuenta con objetivos y recursos educativos en línea. \\
\hline
\end{tabular}

Fuente: Elaboración propia a partir de Aragón, et.al. (2017) y CODAES (2015)

Por otro lado, el docente y el alumno son considerados participantes del proceso de enseñanza-aprendizaje en la educación en línea o virtual. Cada uno desempeña un papel importante, además deben desarrollar actividades, tener interacciones significativas y realizar trabajo colaborativo de manera lineal (entre pares) o vertical (docente-alumno).

El diseño sólido facilita la instrucción efectiva, eficiente, atractiva, interactiva y divertida dentro de los Entorno Virtual de Aprendizaje (EVA). Cuando el conocimiento existente es el abastecedor de uno nuevo, este se encuentra inmerso en el mundo del estudiante y él o ella descubren su gran capacidad para adquirir su propio aprendizaje; inclusive, si el instructor se apoya de las herramientas tecnológicas para el aprendizaje, propicia el desarrollo de habilidades cognitivas complejas (Khadimally, 2015). 
El curriculum es otro componente importante. Para Petrina (2007) existe una diferencia entre diseño curricular e instruccional, donde se muestra una relación en la creación de contenidos educativos y las formas de estos contenidos, respectivamente; determina su prioridad en la teoría curricular sobre el qué se debe aprender, mientras que la del diseño instruccional identifica el cómo se debe organizar para enseñar.

Además, Molina Avilés y Molina Avilés (2005) aseguran que los contenidos curriculares y sus objetivos son elementos que requiere el diseño instruccional para llevar a cabo el proceso educativo de enseñanza-aprendizaje. Por ello, la interdisciplinariedad es un componente importante en la relación educación y tecnología, pues esta permite la innovación de la enseñanza en las Instituciones de Educación Superior (IES), siendo esta enseñanza conformada por estrategias, procesos y tareas prácticas.

Por otro lado, Pukelis (2011) distingue la habilidad como un prerrequisito para la destreza, mientras que la destreza se demuestra por medio del conocimiento, saber-hacer, la comprensión y las habilidades. En otras palabras, la destreza es la habilidad articulada conforme a requerimientos específicos (experiencia práctica o investigaciones científicas); de esta forma, Tuning Guide (2010) propone que los programas de estudios se diseñen en términos de productos de aprendizaje (Purkelis, 2011).

Es necesario destacar que los materiales didácticos deben promover el aprendizaje, a través de actividades colaborativas que desarrollen y demuestren la metacognición; a través de la tecnología educativa se permite la mediación entre el alumno, el docente y la interacción con los materiales. La creación de estos materiales debe establecer una relación sencilla entre el EVA y la comunicación dentro del entorno con los participantes (Londoño, E., 2011). Los materiales elegidos deben estar centrados en los objetivos, los estudiantes, los aprendizajes y el contexto para que de esta forma funcionen solo como guía en el logro del aprendizaje en red, propiciando la retroalimentación que demuestre el conocimiento construido entre todos los participantes.

En síntesis, sería oportuno que las IES consideren la creación de un diseño instruccional bajo el enfoque del conectivismo como una nueva opción en el proceso educativo, puesto que una ecología de aprendizajes sólida implica orientar los contenidos curriculares con los recursos didácticos mediados por la tecnología educativa, para permitir su adecuada organización en la enseñanza y, de esta manera, facilitar la evidencia de los aprendizajes; inclusive, puede fortalecer la destreza de literacidad informacional en sus participantes, tan requerida por la sociedad del conocimiento. Además, la correcta aplicación de las herramientas tecnológicas con el manejo de la información para facilitar el intercambio del conocimiento a través de los de las diferentes conexiones, consolida el aprendizaje en redes.

\section{LA UNIVERSIDAD EN MÉXICO Y SUS PERSPECTIVAS}

De ante mano, se confirma que los cambios sociales, económicos y laborales obligan a las IES a reformar los modelos pedagógicos y educativos, así como las habilidades didácticas de los docentes, al igual que las experiencias de aprendizaje de los alumnos. Es por ello que, para la Asociación Nacional de Universidades e Instituciones de Educación Superior (ANUIES) las TIC tran- 
sitarán hacia las Tecnologías para el Aprendizaje y el Conocimiento (TAC) (ANUIES, 2018) en los diferentes espacios (formales e informales) y modalidades educativas (presenciales, abiertos, a distancia y dual) para configurar programas educativos flexibles, con accesibilidad y de calidad, donde el alumno será el centro del proceso enseñanza-aprendizaje, vivenciando un aprendizaje activo en su formación de competencias del siglo XXI; apoyada de la investigación como fundamentación de dicha formación, donde las IES aportan favorablemente al desarrollo del entorno.

De acuerdo con Lupion y Rama (2010), México es uno de los países Latinoamericanos que cuenta con políticas públicas sólidas que promueven la educación a distancia en el sector público, además el costo de esta oferta pública educativa es muy baja o casi gratuita. En las últimas dos décadas del siglo pasado las políticas gubernamentales para la educación superior se enfocaron hacia el ámbito económico y de globalización, destacando su inclusión en programas sectoriales y proyectos estratégicos para atender las demandas de la población juvenil (Amador, 2010).

En el Plan Sectorial de Educación 2013-2018 se destaca el aprovechamiento de las TIC para fortalecer la educación media y superior, mediante su incorporación a planes y programas de estudios, nuevos procesos de enseñanza, capacitación docente y profesional, entre otros (SEP, 2013). Apoyando lo anterior, la Estrategia Digital Nacional (2013) pretende la digitalización en México, gracias a sus cinco objetivos y habilitadores, resaltando los que involucran la transformación del modelo educativo y conectividad, respectivamente (Estrategia Digital Nacional, 2013). A pesar de los esfuerzos realizados por el gobierno mexicano para acercar las TIC a su población, ANUIES (2018) señala que México, con relación al resto del mundo, ocupa el lugar 82 en cuanto al acceso a Internet en las escuelas, solo el $47 \%$ de los hogares cuenta con internet y los estados más rezagados con Chiapas y Oaxaca con un $13.3 \%$.

Además, ANUIES (2018) hace referencia a la cuarta revolución industrial (revolución 4.0) que no sólo consiste en el uso de nuevos recursos tecnológicos y sistemas inteligentes interconectados, su alcance va más allá y aún se desconocen sus impactos sociales; en este sentido, se debe impulsar el desarrollo de nuevos modelos educativos, consiguiendo llamarla educación 4.0 que responde a los desafíos de la nueva época y haciendo factible una aproximación a la sociedad y la economía del conocimiento. Proponiendo que, en la educación 4.0 "los estudiantes deben adquirir nuevas habilidades de pensamiento, mayor creatividad, capacidad para resolver problemas complejos y tomar decisiones con altos umbrales de incertidumbre, aprender a discriminar, analizar e interpretar grandes volúmenes de información, así como dominar diferentes lenguas extranjeras" (ANUIES, 2018, p. 42).

El conectivismo, que surgió en la era digital, ofrece una explicación a las nuevas formas de aprendizaje y de conocimiento, desarrollados a través de conexiones en redes que aportan al desempeño de los individuos y sus comunidades. Destacando que el flujo de información es continuo y dinámico, lo convierte en conocimiento gracias a las conexiones infinitas de datos en bruto, mientras que el aprendizaje es el reconocimiento, la interacción y retroalimentación del conocimiento con sus conexiones dentro de las redes.

El desarrollo de un diseño instruccional bajo una perspectiva conectivista, correctamente llamada ecología de aprendizajes, ofrece a las IES muestra beneficios como optimización del trabajo docente, materiales educativos favorables al aprendizaje (crear, diseñar, seleccionar y 
evaluar), desarrollo del pensamiento lógico y crítico que fortalece habilidades para la gestión de la información aportando al aprendizaje en redes por los estudiantes.

Por otro lado, las IES requieren actualizar sus planes y programas de estudio mejorando el proceso de enseñanza-aprendizaje, que a su vez facilita a la comunidad educativa su incorporación ciudadana a una vida productiva. La distinción de un modelo pedagógico puede estar dado por el diseño curricular y sus métodos de entrega característicos. La currícula innovadora provee un contexto de integración del aprendizaje, además de consolidar el desarrollo de la facultad, favorece un contexto colaborativo para la misma facultad, por último, permite diferenciar a la institución de sus competidores (Routhieaux, 2015).

En otro sentido, Umaña (2014) asegura que las IES interesadas en solventar los nuevos retos educativos deben revisar periódicamente sus modelos de diseño instruccional considerando las políticas educativas y los contextos sociales en que se encuentran. Por consiguiente, es labor de los diseñadores instruccionales crear ecologías de aprendizajes mediante herramientas tecnológicas que favorezcan el cambio educativo (Rodríguez, y Molero, 2009), además de facilitar y promover las destrezas en los productos de aprendizaje (Purkelis, 2011) de los planes y programas.

Con todo lo antes expuesto, considero pertinente resaltar algunas de las perspectivas con las que universidad del siglo XXI en México debe trabajar para alcanzar los objetivos que establece la sociedad del conocimiento en favor de la educación:

1. La IES deben continuar con la incorporación y aplicación de la tecnología educativa dentro y fuera del aula, siempre visualizando los objetivos de la currícula.

2. El desarrollo de la ecología de aprendizajes debe ser incorporado por las IES para fortalecer el aprendizaje formal y fomentar el informal en el alumno.

3. Una ecología de aprendizajes consolidada propicia el conocimiento, aprendizajes, motivación, interacciones sociales, desarrollo de habilidades cognitivas complejas, gestión y administración de la información, entre otras.

4. La ecología de aprendizajes debe mostrar los productos de aprendizajes desarrollados por los alumnos, permitiéndoles evidenciar sus destrezas y capacidades cognitivas.

5. El uso asertivo de los OER dentro de una ecología de aprendizajes promueve la gestión del conocimiento, desarrolla la auto-organización, incrementa la creatividad, favorece la literacidad informacional, entre otros.

6. La ecología de aprendizajes fomenta el desarrollo de una economía del conocimiento dentro de una IES.

7. El conectivismo es una teoría que facilita la comprensión de las nuevas formas de aprendizajes y el uso actual de la tecnología por parte de los estudiantes, de tal forma que las IES deben considerarlo en sus modelos pedagógicos y currículas.

8. La literacidad de la información es una destreza indispensable en la actualidad, por ello las IES requieren de su consolidación y fortalecimiento, echando mano de la ecología de aprendizajes. 
9. La IES deben reconocer en el diseñador instruccional como el facilitador del lenguaje y comprensión de la tecnología educativa para el docente, así como el alumno.

10. El intercambio de conocimientos y aprendizajes en red entre las IES incrementan la economía del conocimiento de la sociedad del conocimiento participando en el desarrollo de la ciencia y la tecnología.

11. Al reconocer el rezago que existe respecto la conectividad y accesibilidad de las tecnologías digitales para la población, obliga a las IES a colaborar con las instituciones gubernamentales encargadas con el desarrollo de políticas públicas o investigaciones que acorte la brecha digital en la educación y, por ende, en la sociedad.

\section{REFERENCIAS}

Amador, R. (2010). La Educación Superior a Distancia en México. Realidades y tendencias. En P. Lupion, y C. Rama. (coords.). (2010). La Educación Superior a Distancia en América Latina y el Caribe. Realidades $y$ tendencias. (pp.129-144). UNISUL. Recuperado de https://bit.ly/2ATw1G1

Alismail, H. y McGuire, P. (2015). 21st century standards and curriculum: current research and practice. Journal of Education and Practice 6 (6). Recuperado de https://bit.ly/2TBZgDT

Asociación Nacional de Universidades e Instituciones de Educación Superior [ANUIES]. (2018). Visión y acción 2030 propuesta de la ANUIES para renovar la educación superior en México. ANUIES. Recuperado de https://bit.ly/3d19xBk

Aragón, E., Aguilar, M. y Navarro, J. (2017). Sistema instruccional de apoyo a la enseñanza del sentido numérico. Revista de educación (375), pp. 375-333. Doi: DOI: 10.4438/1988-592X-RE-2016-375-333

Barojas, J., Sierra, J. y Martínez, R. (2006). Gestión del conocimiento en un programa multidisciplinario de educación a distancia. Virtual Educa. Recuperado de https://bit.ly/2LVhbkL

Bartolomé, A. (2011). Conectivismo: aprender em rede e na rede. En B. Carneiro Leão, M.: Tecnologias na Educaçao: Uma abordagem crítica para uma atuaçao práctica. Recife (Brasil): UFRPE. pp. 71-86. Recuperado de https://bit.ly/2zqFe8x

Comisión Interamericana de Telecomunicaciones (2003). Agenda de conectividad para las Américas plan de acción de Quito. (25 noviembre 2003). Organización de los Estados Americanos [OEA]. Recuperado de https://bit.ly/2TysOSX

Comunidades Digitales para el Aprendizaje en la Educación. [CODAES]. (2015). Modelo de diseño instruccional. Universidad de Colima. Recuperado de https://bit.ly/2ytSfO6

Downes, S. (2012). Connectivism and Connective Knowledge Essays on meaning and learning networks. Creative Common License. Recuperado de https://bit.ly/3gfAQtL

García Aretio, L., Ruíz, M., Quintanal, J., García Blanco, M. y García Pérez, M. (coord.). (2009). Concepción $y$ tendencias de la Educación a Distancia en América Latina. Documentos de trabajo No. 2. Centro de Altos Estudios Universitarios de la OEI.

García Cabrero, B., Márquez, L., Bustos, A., Miranda, G. A. y Espíndola, S. (2008). Análisis de los patrones de interacción y construcción del conocimiento en ambientes de aprendizaje en línea: una estrategia metodológica. Revista Electrónica de Investigación Educativa 10 (1). Recuperado de https:// bit.ly/2ZDRCN8 


\section{EST U D I S Y E N S Y O S}

Estrategia digital nacional 2013-2018. Gobierno de la República. (2013). Recuperado de https://bit. ly/2A6a $6 \mathrm{Lu}$

Gómez, J. (2013). Importancia de los procesos comunicativos como intervención pedagógica y uso de herramientas tecnológicas: un análisis desde el diseño instruccional. En Católica del Norte Fundación Universitaria. El diseño instruccional: reflexiones y perspectivas en la Católica del Norte Fundación Universitaria. (pp. 93-118). Recuperado de https://bit.ly/36tSUvK

Gutiérrez, L. (2012). Conectivismo como teoría de aprendizaje: conceptos, ideas, y posibles limitaciones. Educación y Tecnología, (1), pp. 111-122. Recuperado a partir de https://bit.ly/2zriGEE

Khadimally, S. (2015). Designing Effective Curricula with an Interactive Collaborative Curriculum Design Tool (CCDT). Turkish Online Journal of Educational Technology TOJET. 14(3), pp. 32-62. Recuperado de https://bit.ly/3cY6k5y

Keeler, C. (2008). When Curriculum and Technology Meet: Technology Integration in Methods Courses. Journal of Computing in Teacher Education 25 (1), pp. 23-30. Recuperado de https://bit.ly/3d1iWJa

Londoño, E. (2011). El diseño instruccional en la educación virtual: más allá de la presentación de contenidos. Revista Educación y Desarrollo Social 5(2), pp. 112-127. Recuperado de https://bit. ly/2APPkQz

Lupion, P. y Rama, C. (2010). Algunas de las características dominantes de la educación a distancia en América Latina y el Caribe. En Lupion, P. y Rama, C. (Coord.). (2010). La educación superior a distancia en América Latina y el Caribe realidades y tendencias. (pp. 9-16). Editorial UNISUL. Recuperado de https://bit.ly/2ATw1G1

Molina Avilés, M. y Molina Avilés, J. (2005). Fundamentos teóricos de la educación a distancia diseño instruccional para el aprendizaje significativo. Trabajo presentado en el Encuentro Internacional de Educación Superior Virtual Educa 2005. Recuperado de https://bit.ly/2zsyxmf

Oficina Regional de Educación para América Latina y el Caribe. [OREALC]. (2013). Enfoques estratégicos sobre las TIC en la educación en América Latina y el Caribe. Chile: Organización de las Naciones Unidas para la Educación, la Ciencia y la Cultura [UNESCO]. Recuperado de https://bit.ly/3gmplAq

Organización de las Naciones Unidas para la Educación, la Ciencia y la Cultura [UNESCO]. (2010). Conferencia Mundial sobre la Educación Superior - 2009: La nueva dinámica de la educación superior y la investigación para el cambio social y el desarrollo (Informe ED.2009/CONF.402/2). UNESCO. Recuperado de https://bit.ly/2zrxyCX

Organización de las Naciones Unidas para la Educación, la Ciencia y la Cultura [UNESCO]. (2016). Educación 2030: Declaración de Icheon y marco de acción para la realización del objetivo de desarrollo sostenible 4. (Informe ED-2016/WS/28). UNESCO. Recuperado de https://bit.ly/2zrFtAe

Organización de las Naciones Unidas para la Educación, la Ciencia y la Cultura [UNESCO] (2018). Sociedades del conocimiento: el camino para construir un mundo mejor [Blog]. Recuperado de https:// bit.ly/3doQIhC

Organization for Economic Co-operation and Development [OECD]. (2015). E-Learning in Higher Education in Latin America, Development Centre Studies. OECD Publishing. http://dx.doi. org/10.1787/9789264209992-en

Ovalles, L. (2014). Conectivismo, ¿un nuevo paradigma en la educación actual? Mundo FESC, 4(7), pp. 72-79. Recuperado de https://bit.ly/36strTb 


\section{ESTUDIOS Y ENSAYOS}

Petrina, S. (2007). Curriculum and instructional design. Advance teaching method for the technology classroom. (pp. 251-278). Information Science Publishing.

Pukelis, K. (2011). Studijų programų rengimas iratnaujinimas: studijų rezultatų paradigma. Aukštojo mokslo kokybe (8), pp. 38-73. Recuperado de https://bit.ly/2X02QKf

Rodríguez, A. y Molero, D. (2009). Conectivismo como gestión del conocimiento. REDHECS: Revista electrónica de Humanidades, Educacióny Comunicación Social, 4 (6), pp. 73-85. Recuperado de https:// bit.ly/3cZkoeR

Roldán, N. (2013). De diseños instruccionales a mediadores pedagógicos: apuntes sobre la eficacia de los recursos. En Católica del Norte Fundación Universitaria. El diseño instruccional: reflexiones y perspectivas en la Católica del Norte Fundación Universitaria. (pp. 79-92). Fundación Universitaria Católica del Norte. Recuperado de https://bit.ly/36tSUvK

Routhieaux, R. (2015). Fostering integrated learning and faculty collaboration through curriculum design: a case study. Journal of Curriculum and Teaching 4 (1), pp. 122-132. Doi: http://dx.doi. org/10.5430/jct.v4n1p122

Secretaría de Educación Pública [SEP]. (2013). Programa sectorial de educación 2013-2018. Diario oficial de la Federación [DOF]. Recuperado de https://bit.ly/2X2f459

Siemens, G. (2004). Conectivismo: Una teoría de aprendizaje para la era digital. Traducción de Leal, D. (2007) [Blog]. Recuperado de https://bit.ly/2BZUbPL

Siemens, G., (Enero, 2008). Learning and knowing in networks: changing roles for educators and designers. Trabajo presentado en el Instructional Technology Forum [ITFORUM]. pp. 1-26. Recuperado de https://bit.ly/3c1NIAd

Vadillo, G. (2011). Entrevista a George Siemens Desarrollador del Conectivismo. Revista Mexicana de Bachillerato a Distancia 11 (21), pp. 41-47. Doi: http://dx.doi.org/10.22201/cuaed.20074751e.2011.6

Villalonga, A. (2015). La Educación Superior a distancia modelos, retos y oportunidades. Oficina Regional de Cultura para América Latina y el Caribe. Oficina de la UNESCO la Habana. Recuperado de https:// bit.ly/2yyvwR6

Umaña, A. (2014). Evaluación de modelos de diseño instruccional: una revisión de literatura.Innovaciones Educativas, (21), pp. 23-30. Recuperado de https://bit.ly/2A20HVp 